\title{
Pemphigus vulgaris: the other half of the story
}

\author{
Richard S. Kalish
}

Department of Dermatology, Health Sciences Center T-16, Room 060,

State University of New York at Stony Brook, Stony Brook, New York 11794-8165, USA.

Phone: (631) 444-3843; Fax: (631) 444-3844; E-mail: rkalish@epo.som.sunysb.edu.

Pemphigus vulgaris pathogenesis had been considered well understood and was in danger of being "finished," as was physics at the end of the 19th century. With the publication in this issue of a paper by Nguyen and colleagues (1), the understanding of pemphigus pathogenesis must undergo a major revision. This turning point brings with it the promise of a more complete picture, as well as new therapeutic approaches for this serious disease.

\section{Pemphigus vulgaris and pemphigus foliaceous}

Pemphigus vulgaris (PV) is a chronic autoimmune blistering disease often lasting many years (2). Prior to use of corticosteroids, PV mortality approached $100 \%$. Currently, with immunosuppressive therapy, death from pemphigus is unusual, but morbidity and mortality from chronic corticosteroid use are considerable, and additional therapies are needed. The blisters in PV are characterized by an intraepidermal split above the basal keratinocytes. Suprabasal keratinocytes lose adhesion and round up in a process known as acantholysis. On direct immunofluorescence, IgG is seen in an intercellular distribution within the epidermis. The disease is mediated by antibodies and can be transferred to neonatal mice by the injection of IgG (3); injected mice exhibit blisters with typical histology, as well as positive direct immunofluorescence for intercellular IgG in the epidermis. PV IgG is also able to induce acantholysis in culture, with loss of adherence of keratinocyte monolayers.

The mechanism by which PV IgG induces acantholysis has been subject to dispute. However, it has become dogma that the autoantigen responsible for PV is desmoglein 3 (Dsg3), a $130-\mathrm{kDa}$ desmosomal adhesion molecule (4). Autoantibody to Dsg3 appears to be the unifying feature of PV in patients with detectable circulating antibodies, although, as discussed below, autoantibody profiles differ among PV patients, and sera from some individuals contain an additional activity to a distinct, $160-\mathrm{kDa}$ desmosomal protein, desmoglein 1 (Dsg1) (4). In pemphigus foliaceous $(\mathrm{PF})$, a variant of pemphigus that is associated with autoantibodies to Dsg1, the split occurs higher in the epidermis at sites below the granular layer.

\section{The desmoglein compensation hypothesis}

Desmogleins 1 and 3 are members of the cadherin family of adhesion molecules. They are transmembrane proteins that interact with plakoglobin, a component of the dense plaque of the desmosome. It is believed that antibodies to Dsg3 and Dsg1 directly induce acantholysis by interfering with the adhesive function of these target molecules. The classic demonstration that anti-Dsg3 antibodies cause PV rests on the ability of recombinant Dsg3 to absorb out pathogenic activity from PV serum (5). However, this work employed not the native Dsg3 protein per se, but rather a recombinant fusion protein composed of Dsg3 and the constant region of human IgG1 (Dsg3-Ig). Absorption of PV sera with Dsg3-Ig prevents both induction of blisters and acantholysis following transfer to neonatal mice. Similar work with recombinant Dsg1-Ig shows that this chimeric protein can absorb out the pathogenicity of PF antibodies (6).

The different locations of epidermal separation seen in PV and in PF can be explained by the distribution of Dsg3 and Dsg1, further supporting the role of these autoantigens in pemphigus. Because the two desmogleins apparently compensate for each other's absence to prevent acantholysis (7), PF sera (containing antibodies to Dsg1 but not Dsg3) can disrupt the epidermis only at sites where Dsg1 is expressed exclusively; conversely, PV sera that contain antibodies to Dsg3 but not Dsg1 can disrupt the epidermis only at sites where Dsg3 is expressed exclusively. Interestingly, the distribution of these two proteins differs between epidermal tissues (Figure 1). Thus, in the skin, Dsg1 is expressed without Dsg3 in the upper levels of the epidermis, the site where blisters occur in PF. Mucous membranes differ in that Dsg3 is expressed throughout the epidermis, whereas Dsg1 is restricted to the upper levels of the epidermis. For this reason, PF serum is not associated with blisters of mucous membranes, since Dsg3 is also present and can maintain the structure of the mucosal epidermis even in the presence of blocking antibodies to Dsg1. However, PV serum is associated with suprabasal blisters of mucous membranes, where Dsg3 is present but Dsg1 is absent.

Blisters in the skin are found in some but not all individuals with PV and occur at the suprabasal layer, where keratinocytes express both Dsg3 and Dsg1 (Figure 1). As predicted by the desmoglein compensation hypothesis, PV of skin is associated with the simultaneous presence of anti-Dsg3 and anti-Dsg1 (8). PV patients with antiDsg3 alone exhibit mucous membrane lesions (mucosal PV), but their skin is not blistered. Anti-Dsg1 antibodies in PV sera are pathogenic and can induce skin blisters in neonatal mice (9). Mahoney, Stanley, and colleagues have further developed and confirmed the desmoglein compensation hypothesis (7), showing that antibodies to both Dsg3 and Dsg1 are required to induce skin blisters in wild-type neonatal mice. Using Dsg 3 knockout mice, these authors showed that Dsg1 can compensate for the lack of Dsg3 but that PF sera (containing anti-Dsg1 antibodies) can induces blisters in the mutant animals' oral mucosa, a tissue that is normally spared because of the presence of Dsg3. 
Figure 1

Localization of split in PV and PF is explained by the desmoglein compensation hypothesis. In skin (upper panels), Dsg3 is expressed at lower levels of the epidermis, whereas Dsg1 occurs at upper levels. In mucous membranes (lower panels), Dsg3 is present throughout the epidermis, and Dsg1 is present only at upper levels. In skin, anti-Dsg1 induces a split below the granular layer, where Dsg3 is lacking. In the oral mucosa, anti-Dsg3 alone is sufficient to induce suprabasal blisters, since Dsg1 is lacking at lower levels of the epidermis. However, the suprabasal blisters of skin in PV patients are associated with antibodies to both Dsg3 and Dsg1. Figure adapted from Mahoney et al. (7).

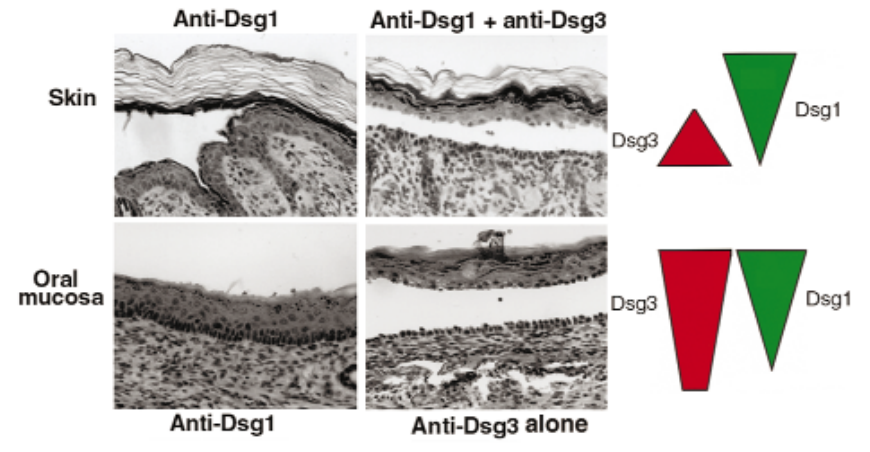

\section{A pathological role for other autoantigens}

These studies provide strong confirmation that Dsg1 and Dsg3 are etiologically relevant autoantigens in PV and PF. Remarkably, however, Nguyen et al. (1) now report that antibodies from PV serum can induce blisters even in Dsg3deficient mice. Crucially, the authors determined that these PV antibodies contain anti-Dsg3 activity but no antiDsg1 activity. Therefore, although Dsg1 might be thought to compensate for the lack of Dsg3 in these mutant animals, the effect of the PV antibodies cannot be ascribed to a blockade of Dsg1. The authors confirmed their results using both $D s g 3$-targeted knockout mice and a spontaneous mutant mouse strain, $D$ sg3 $3^{b a l} / D s g 3^{b a l}$ mice, which carries a null mutation in $D s g 3$.

Nguyen and coworkers' evidence for a Dsg3-independent effect of PV antibodies (1) is unexpected, but other findings in the current report provide a basis for understanding this effect. The authors probed immunoblots of epidermal extracts using these PV sera and detected multiple nondesmoglein antigens. This finding in itself might have been expected, since additional bands are visible on published immunoblots (10) but have been generally ignored. Prior to the cloning of desmoglein antigens, similar analyses of patients' sera showed evidence of additional PV autoantigens, including one still-uncharacterized protein of $33 \mathrm{kDa}$ (11). More surprising is the finding of Nguyen et al. that the antibody eluted from a Dsg3-Ig column, which induces blisters in Dsg3-deficient mice, is also nonspecific and recognizes multiple nondesmoglein antigens in addition to Dsg3.

The classic demonstration that absorption of PV sera with recombinant Dsg3 removes pathogenicity for transfer to neonatal mice (5) must therefore be reinterpreted in light of the current evidence that the antibodies absorbed can bind multiple antigens in addition to Dsg3. This absorption is not effective unless the Dsg3 preparation used is a recombinant fusion protein with Ig (Dsg3-Ig). Recombinant Dsg3 that is not produced as a fusion protein with Ig fails to absorb out pathogenic activity of PV sera. While this finding has been explained by postulating that the Ig sequence alters the conformation of the Dsg3 in the fusion protein, the present evidence that Dsg3Ig affinity columns are nonspecific suggests a simpler explanation: autoantibodies specific for other epidermal autoantigens, distinct from Dsg3, associate with Dsg3-Ig, and some of these antibodies may contribute to the blistering seen in neonatal mice receiving PV sera (1). Previous researchers had not examined the eluted antibodies to confirm that they were monospecific for the desmoglein.

It is possible that the Dsg3-Ig column is nonspecific because of the presence in PV serum of rheumatoid factor, an IgG-binding protein that could crosslink Dsg3-Ig on the column with nonspecific IgG in the serum sample. Alternately, Nguyen et al. propose that the Dsg3-Ig fusion protein itself contains cross-reactive epitopes (1). It should be noted that the absorption experiments on PF serum employed an analogous construct, Dsg1-Ig, to test for the involvement of anti-Dsg1 antibodies in that disorder (6), and that there was no evidence reported to indicate that the absorbed antibody was specific for Dsg1. Hence, although the molecular basis for the lack of specificity is still uncertain, nondesmoglein antigens clearly play a role in PV and possibly in $\mathrm{PF}$ as well.

\section{Intracellular mechanisms of acantholysis}

Induction of acantholysis is an active process that appears to be more complex than the simple interaction of antibodies with adhesion molecules (12). Signaling events associated with binding of PV sera to keratinocytes include activation of phospholipase C, resulting in increases in both inositol 1,4,5-trisphosphate $\left(\mathrm{IP}_{3}\right)$ and diacylglycerol (DAG). There is an increase in intracellular calcium, which appears to result from $\mathrm{IP}_{3}$-mediated release of intracellular stores. Protein kinase $\mathrm{C}$ (PKC) is also activated, presumably by DAG. Dsg3 is phosphorylated by a kinase other than PKC and appears to disassociate from plakoglobin, another component of the dense plaque of the desmosome. This dissociation may explain the ability of PV sera to deplete Dsg3 from desmosomes (13).

The changes of keratinocyte intracellular calcium induced by PV sera are similar to those seen on stimulation of these cells with muscarinic agonists (14). Human keratinocytes express functional cholinergic receptors of both nicotinic and muscarinic classes $(14,15)$, and stimulation of these receptors profoundly alters adhesive interactions, at least in part by regulating intracellular calcium levels. Both cholinergic receptors stimulate keratinocyte adhesion in culture, although their mechanisms of action are distinct. Nicotinic agonists induce calcium influx, while muscarinic agonists increase intracellular calcium by release of intracellular stores. Antagonists of nicotinic receptors (e.g., mecamylamine) and muscarinic receptors (e.g., atropine) induce keratinocyte detachment and acantholysis in vitro.

Autoantibodies to keratinocyte cholinergic receptors, including two 
that were recently cloned by the Grando laboratory, can be detected in most PV patients (16). Pemphaxin is a keratinocyte PV autoantigen with annexin homology, and the ability to bind acetylcholine (17). The molecule is a homodimer composed of 40-kDa subunits. Antibody to pemphaxin induces acantholysis of keratinocytes in culture, and preabsorption of PV sera with pemphaxin depletes pathogenicity for neonatal mice. However, the eluted material is not pathogenic, suggesting that this autoantibody is necessary but not sufficient. Corticosteroids boost the expression of pemphaxin, which may be relevant to the therapeutic effect of corticosteroids on acantholysis in PV (18). Keratinocytes also express a unique cholinergic receptor that binds both nicotinic and muscarinic agonists (19). This $50-\mathrm{kDa} \alpha 9$ acetylcholine receptor is recognized by sera from PV patients as an autoantigen. Antibody to $\alpha 9$ acetylcholine receptor binds human epidermis in an intercellular pattern. This antibody induces acantholysis of human keratinocytes in vitro but does not induce blisters in vivo upon transfer to newborn mice. It is hypothesized that $\alpha 9$ acetylcholine receptor and pemphaxin are among several acetylcholine receptor autoantigens in $\mathrm{PV}$.

\section{A unifying hypothesis}

Grando and colleagues have proposed that blistering in PV is the result of synergy between anti-desmoglein antibodies and anti-cholinergic receptor antibodies (1). Antibodies to keratinocyte cholinergic receptors induce acantholysis, in this view, while antibodies to Dsg3 deplete Dsg3 from desmosomes. It is hypothesized that both classes of antibodies are required to induce blisters in humans or wild-type mice. As seen in the current report (1), nondesmoglein antibodies alone are sufficient to induce blisters in Dsg3-deficient mice. Depletion of anti-cholinergic antibodies impairs the ability of PV serum to induce blisters in mice, but anti-cholinergic receptor antibodies do not induce blisters in normal mice in the absence of anti-desmoglein antibodies (17). Development of PV is further proposed to require epitope spreading from Dsg3 and Dsg1 to keratinocyte cholinergic receptors. Consistent with this model, Brandsen et al. (20) have detected anti-Dsg3 antibodies, along with positive direct immunofluorescence of skin, in apparently healthy first-degree relatives of $\mathrm{PV}$ patients (20).

\section{Directions for further research}

The work of Nguyen et al. (1) raises a number of crucial questions about the pathogenesis of blistering in PV and PF. First, what are the relevant nondesmoglein autoantigens of PV? The $\alpha 9$ acetylcholine receptor and pemphaxin clearly are promising candidates, but direct evidence is still lacking for synergy between antibodies to desmogleins and antibodies to either of these receptors. Second, how is acantholysis induced by antibody to these antigens? The molecular events occurring at the desmosome and elsewhere in the keratinocyte still need to be defined if we are to understand the PV pathogenesis or design drug therapies - possibly involving cholinergic agonists (1) - to preserve epidermal structure. Finally, there is a series of questions about dysregulation of immune responses that might be asked of other autoimmune disorders as well: How does disease arise? How is tolerance lost? How do antibody responses spread, in this case between the desmoglein epitopes and those in other relevant autoantigens? With better definition of autoantigens, it may be possible to develop antigenspecific immune modulation, such as immune deviation or induction of tolerance, to prevent or even reverse these pathological responses.

\section{Acknowledgments}

This work was partially supported by NIH grant 1PO1NS3409201A2. In honor of the 65th birthday of Stuart F. Schlossman (Dana-Farber Cancer Institute, Boston, Massachusetts, USA), I would like to acknowledge the assistance he provided to me when I was a research fellow at the start of my career.

1. Nguyen, V.T., Ndoye, A., Shultz L.D., Pittelkow, M.R., and Grando, S.A. 2000. Antibodies against keratinocyte antigens other than desmogleins 1 and 3 can induce pemphigus vulgaris-like lesions. J. Clin. Invest. 106:1467-1479.

2. Herbst, A., and Bystryn, J.C. 2000. Patterns of remission in pemphigus vulgaris. J. Am. Acad. Dermatol. 42:422-427.

3. Anhalt, G.J., Labib, R.S., Voorhees, J.J., Beals, T.F., and Diaz, L.A. 1982. Induction of pemphigus in neonatal mice by passive transfer of IgG from patients with the disease. N. Engl. J. Med. 306:1189-1196

4. Diaz, L.A., and Giudice, G.J. 2000. End of the century overview of skin blisters. Arch. Dermatol. 136:106-112.
5. Amagai, M., Hashimoto, T., Shimizu, N., and Nishikawa, T. 1994. Absorption of pathogenic autoantibodies by the extracellular domain of pemphigus vulgaris antigen (Dsg3) produced by baculovirus. J. Clin. Invest. 94:59-67.

6. Amagai, M., Hashimoto, T., Green, K.J., Shimizu, N., and Nishikawa, T. 1995. Antigenspecific immunoadsorption of pathogenic autoantibodies in pemphigus foliaceus. J. Invest. Dermatol. 104:895-901.

7. Mahoney, M.G., et al. 1999. Explanations for the clinical and microscopic localization of lesions in pemphigus foliaceus and vulgaris. J. Clin. Invest. 103:461-468.

8. Ding, X., et al. 1997. Mucosal and mucocutaneous (generalized) pemphigus vulgaris show distinct autoantibody profiles. J. Invest. Dermatol. 109:592-596.

9. Ding, X., Diaz, L.A., Fairley, J.A., Giudice, G.J., and Liu, Z. 1999. The anti-desmoglein 1 autoantibodies in pemphigus vulgaris sera are pathogenic. J. Invest. Dermatol. 112:739-743.

10. Hashimoto, T., Ogawa, M.M., Konohana, A., and Nishikawa, T. 1990. Detection of pemphigus vulgaris and pemphigus foliaceus antigens by immunoblot analysis using different antigen sources. J. Invest. Dermatol. 94:327-331.

11. Peterson, L.L., and Wuepper, K.D. 1984. Isolation and purification of a pemphigus vulgaris antigen from human epidermis. J. Clin. Invest. 73:1113-1120

12. Kitajima, Y., Aoyama, Y., and Seishima, M. 1999. Transmembrane signaling for adhesive regulation of desmosomes and hemidesmosomes, and for cell-cell detachment induced by pemphigus IgG in cultured keratinocytes: involvement of protein kinase C. J. Investig. Dermatol. Symp. Proc. 4:137-144.

13. Aoyama, Y., and Kitajima, Y. 1999. Pemphigus vulgaris-IgG causes a rapid depletion of desmoglein 3 (Dsg3) from the triton X-100 soluble pools, leading to the formation of Dsg3depleted desmosomes in a human squamous carcinoma cell line, DJM-1 cells. J. Invest. Dermatol. 112:67-71.

14. Grando, S.A. 1997. Biological functions of keratinocyte cholinergic receptors. J. Investig. Dermatol. Symp. Proc. 2:41-48.

15. Grando, S.A., et al. 1995. A nicotinic acetylcholine receptor regulating cell adhesion and motility is expressed in human keratinocytes. $J$. Invest. Dermatol. 105:774-781.

16. Nguyen, V.T., et al. 1998. The pathophysiological significance of nondesmoglein targets of pemphigus autoimmunity. Development of antibodies against keratinocyte cholinergic receptors in patients with pemphigus vulgaris and pemphigus foliaceus. Arch. Dermatol. 134:971-980.

17. Nguyen, V.T., Ndoye, A., and Grando, S.A. 2000. Pemphigus vulgaris antibody identifies pemphaxin. A novel keratinocyte annexin-like molecule binding acetylcholine. J. Biol. Chem. 275:29466-29476.

18. Nguyen, V., Ndoye, A., and Grando, A. 1999. A pool of disease-causing pemphigus vulgaris IgGs contains an autoantibody to pemphaxin, a calcium-dependent keratinocyte annexin-like molecule that can be upregulated by methylprednisolone. J. Invest. Dermatol. 112:564. (Abstr.)

19. Nguyen, V.T., Ndoye, A., and Grando, S.A. 2000 Novel human alpha9 acetylcholine receptor regulating keratinocyte adhesion is targeted by pemphigus vulgaris autoimmunity. Am. J. Pathol. 157:1377-1391.

20. Brandsen, R., et al. 1997. Circulating pemphigus IgG in families of patients with pemphigus: comparison of indirect immunofluorescence, direct immunofluorescence, and immunoblotting. J. Am. Acad. Dermatol. 36:44-52. 